\title{
CREATION OF PARTICLES IN COSMOLOGY
}

\author{
Ya. B. ZEL'DOVICH \\ Institute of Applied Mathematics, USSR Academy of Sciences, Moscow, U.S.S.R.
}

The creation of particles is a process which can only be described by quantum field theory. The old classical theories dealing with indestructible particles are incompatible with particle creation. It was the discovery of the corpuscular nature of light (Einstein, 1905) and the prediction of antiparticles (Dirac, 1929) which demonstrated that particle creation was possible. The creation of particles may influence the cosmological equations through the energy-stress tensor of these particles. For physical cosmology the particles themselves are important. Therefore this report deals with a particular example of the impact of quantum mechanics on cosmological theory.

Many papers have dealt with quantum corrections to the gravitational field equations - see for example Ginzburg et al. (1971) and the papers mentioned therein. But these corrections were of a type which modified the elasticity of space-time. The equations

$$
\frac{c^{4}}{8 \pi G}\left(R_{i k}-\frac{1}{2} g_{i k} R\right)=T_{i k}
$$

can be interpreted in terms of the left-hand side being the elasticity and the right-hand side being the force which tries to make space-time curved.

The quantum-corrections modify (1) in the following way:

$$
\begin{aligned}
& \frac{c^{4}}{8 \pi G}\left(R_{i k}-\frac{1}{2} g_{i k} R\right)+\beta\left(R_{i k}-\frac{1}{2} g_{i k} R\right)+ \\
& +\gamma\left(R_{i l k m} R^{l m}+\cdots\right)+\cdots+\alpha g_{i k}=T_{i k} .
\end{aligned}
$$

The term $\alpha g_{i k}$ is the effective cosmological term; it can, in principle, be different from zero. As was shown in the paper by Petrosian, at this symposium, modern observational data make it possible to impose strong upper and lower limits on the value of the cosmological constant. The observed gravitational constant is

$$
G=G_{0}\left(1+\frac{8 \pi \beta G_{0}}{c^{4}}\right)^{-1}
$$

(renormalisation of $G$ ) and new terms of higher order occur. The general property of (2) is that when the curvature is small the equations are those of standard general relativity, independent of their previous history.

So much for the earlier approach.

The new approach to quantum corrections, developed in this report, is equivalent to introducing viscosity into space-time. The additional energy-stress components are proportional to time derivatives of the curvature.

After a closed-loop process or after a singularity some irreversible changes occur - the created particles do not disappear, even after the curvature becomes small. So 
there exists a qualitative difference between viscosity and elasticity, i.e. between particle creation and vacuum polarisation.

An important point is that the rest mass of particles is small $\mathrm{Gm}^{2} / \hbar c \sim 10^{-37}-10^{-43}$, and some particles even have $m \equiv 0$ (photons, neutrinos). The approximation $m=0$ for all particles is therefore appropriate. But an ultrarelativistic gas (consisting of massless fields and particles) has an important general property: it has shear viscosity, but no bulk expansion viscosity (the so-called second viscosity is zero). This property remains valid for vacuum viscosity due to particle creation.

The most important result is that Friedmann type cosmological models give no appreciable particle creation even in the singularity. This was shown by the calculations of Parker (1969). The general principle of Weyl's conformal invariance underlying this result, was emphasized also by Zel'dovich and Starobinsky (1971).

This negative result does not mean that particle creation is not important at all. The general solution of Einstein's equations is highly anisotropic near the singularity (see the report by Belinsky, Lifshitz, and Khalatnikov in this volume).

But these solutions were obtained without quantum effects. What happens if they are taken into account? There are two different situations: (1) cosmological expansion and white holes; (2) cosmological collapse and black holes.

In the second case the singularity is the endpoint of evolution. Far from the singularity all quantum effects (viscosity and elasticity) are small. By dimensional arguments $\delta T_{i k} \sim \hbar t^{-4} c^{3}$. Only the very last stages of collapse, $-10^{-43} \mathrm{~s}<t<0$, are distorted by quantum effects of both types. All the interval $-\infty<t<-10^{-43} \mathrm{~s}$ is unaffected and classical results are valid. Here $10^{-43}=\left(G \hbar / c^{5}\right)^{1 / 2}=t_{p l}$.

The answer is drastically different in the first type of problem concerning the cosmological expansion.

No self-consistent anisotropic solution including particle creation is known, which begins from $t=0$. For a Kasner type metric the equation of energy density is given by

$$
\frac{\mathrm{d} \varepsilon}{\mathrm{d} t}=\frac{\hbar}{c^{3} t^{5}}-\frac{4}{3} \frac{\varepsilon}{t}
$$

This equation has no finite solution for the initial conditions $t=0, \varepsilon=0$.

Let us try to obtain a finite solution using a cut off, $t=t_{1}, \varepsilon=0$. The answer is given by

$$
\begin{aligned}
& \varepsilon=0 ; \quad t<t_{1}, \\
& \varepsilon=\left(t-t_{1}\right) \hbar / c^{3} t_{1}^{5} ; \quad t-t_{1} \ll t_{1}, \\
& \varepsilon=\hbar / c^{3} t_{1}^{8 / 3} t^{4 / 3} ; \quad t \gg t_{1} .
\end{aligned}
$$

But what is important is that particles created near $t_{1}$ do not disappear much later!

In the course of time they will be overwhelmingly important and will cause the Kasner solution to transform into an isotropic expansion. The characteristic time $t_{2}$ of isotropisation is given by

$$
t_{2}=t_{1}^{4} t_{p l}^{-3}
$$

with $t_{p l}=10^{-43} \mathrm{~s}$. 
If $t_{1}$ is pushed back to $t_{p l}$, then so also is $t_{2}$ and the region of existence of the anisotropic solution vanishes.

We conjecture that quantum effects prohibit the most general solutions of the general relativity equations as candidates for the initial cosmological state.

But what remains is not only the Friedmann solution. There is a wider class - the so-called quasi-isotropic solution with arbitrary functions $g_{\alpha \beta}^{(0)}(x)$

$$
\mathrm{d} s^{2}=\mathrm{d} t^{2}-f^{2}(t) \sum_{\alpha, \beta=1,2,3} g_{\alpha \beta}^{(0)} \mathrm{d} x^{\alpha} \mathrm{d} x^{\beta} .
$$

The function $f(t)$ is not arbitrary but depends on the equation of state

$$
f=t^{1 / 2} ; \quad p=\varepsilon / 3
$$

Only the asymptotic solution as $t \rightarrow 0$ is written since the succeeding terms are higher powers of $t$. The solution is obviously nonuniform, $g_{\alpha \beta}^{(0)}$ being functions of space coordinates $x^{\alpha}$.

The solution can be visualized as a Friedmann solution with finite perturbations of the metric. These perturbations include those of scalar type (growing density perturbations) and tensor type (gravitational waves) but not rotational perturbations. All information about the future evolution is given by the functions $g_{\alpha \beta}^{(0)}$.

To summarise this part, anisotropic expansion at the singularity leads to infinite quantum effects and to infinite particle creation. This is considered to prohibit anisotropic singularities. What survives is the quasi-isotropic solution with nonuniform density but without primeaval vortices. These are the first results of the introduction of quantum creation theory into cosmology.

Let us go further and ask if one could explain the creation of particles (the ancestors of the $2.7 \mathrm{~K}$ relic electromagnetic radiation) in the quasi-isotropic solution. A hypothesis of this sort was made by Zel'dovich (1972). The idea of creation from the gravitational field is abandoned because the expansion is isotropic at $t \rightarrow 0$. Instead nonuniformity is introduced which transforms itself into density perturbations and further into acoustical waves, giving heat when they dissipate. To obtain semiquantitative results many detailed assumptions are needed:

(1) The Universe is filled with baryons near the singularity

(2) The equation of state of baryons has the limiting equation of state (Zel'dovich, 1961) at high densities

$$
p=\varepsilon=\hbar^{3} n^{2} / m^{2} c
$$

with $m$ the vector meson mass, of the order of the proton mass, and $n$ the baryon density.

(3) The initial quasi-isotropic solution is of the uniform Friedmann type with superimposed metric perturbations $h_{\alpha \beta}$. These perturbations are finite as $t \rightarrow \infty$, in accord with the results of Lifshitz.

(4) The $h_{\alpha \beta}$ are given by their Fourier spectral decomposition $h_{\alpha \beta}^{(k)}$.

It is assumed that the mean value of the perturbations is independent of scale and 
is of the order of $10^{-4}$. The exact formulation is

$$
\overline{\left(h_{\alpha \beta}\right)^{2}}=\left|h_{\alpha \beta}^{(\kappa)}\right|^{2} \kappa^{3}=10^{-8},
$$

where $\kappa$ is the wave vector and $h(\kappa)$ the Fourier component. These two assumptions (independence of $\kappa$ and absolute magnitude $10^{-4}$ ) are made arbitrarily.

(5) The range of $\kappa$ is taken to be $0<\kappa<\kappa_{\max }=n^{1 / 3}$, where $n$ is the baryon density (see above).

The lower limit, $\kappa \rightarrow 0$ corresponds to very long waves, no limitations on wavelength being made. The upper limit corresponds to setting the shortest wavelength equal to the distance between neighbouring baryons. This corresponds to the idea that the distribution of baryons in space determines the metric and the nonuniform distribution of baryons is the cause of the metric perturbations.

The flat spectrum of metric perturbation corresponds to density perturbations which asymptotically $(t \rightarrow 0$, near the singularity) are given by

$$
\frac{\delta \varrho}{\varrho} \sim \frac{\delta n}{n} \propto t^{4 / 3} n^{-2 / 3} .
$$

Similar perturbations were introduced by Harrison (1970) and by Peebles and $\mathrm{Yu}$ (1970).

We have enumerated comprehensively all the assumptions. In order to relate these to what is physically observable one has to study the evolution of the perturbations.

The ultralong waves $\kappa<10^{-25} n^{-1 / 3}$ (corresponding to $m>10^{18} M_{\odot}$ and to wavelength more than $\lambda=300 \mathrm{Mpc}$ now) remain weak up to the present time. This is in accord with the absence of observable fluctuations $\left(\Delta T / T<10^{-4}\right)$ in the relic radiation.

The moderately long waves (corresponding to masses $10^{15}>M>10^{12} M_{\odot}$ ) have grown to amplitude of the order of unity $(\delta \varrho / \varrho \sim 1)$ in the recent past. It is assumed that these waves are the most important in determining the formation of galaxies, etc. (Doroshkevich et al., this volume, p. 213).

Shorter waves (corresponding to $M<10^{12} M_{\odot}$ ) are dissipated before recombination during the radiation dominated phase or even earlier. They do not leave any visible fingerprints in the density distribution as observed now.

The new point which we should stress is that perturbations, when dissipating, turn their acoustic energy into heat. An order of magnitude calculation shows, that the short-wavelength part of the spectrum assumed above, $\kappa \widetilde{<}_{\kappa_{\max }}$, gives an amount of heat (or more exactly, an amount of entropy) corresponding to what is observed in the relic radiation: $\sim 10^{8}$ photons per baryon, $T \varrho^{-1 / 3} \sim 3 \times 10^{10}$. The tentative conclusion is that a single power law spectrum of perturbations explains two different aspects of our present Universe: first, the macrostructure of the Universe and the relic radiation, and, second, the specific matter entropy (Zel'dovich, 1972).

We have tried to be impartial stressing the arbitrary and unproved parts of the hypothesis. Perhaps we shall see in the near future some approach from first principles to the cosmological singularity. This approach would make superfluous our 
arbitrary assumptions, and this new theory would be of much greater value than ours. Still the possibility of scientific discussion of singularity problems is itself an immense achievement. This possibility is far from being trivial, and is the result of the efforts of many astronomers - optical as well as radio astronomers, of astrophysicists and theoretical physicists.

Note added in proof: Selfconsistent solution for particles and metric see Lukash and Starobinsky, Zh. Eksp. Teor. Fiz. 66, 1515 (1974). The gravitons although massless are not conformally invariant : important consequences for cosmology see Grishchuk, ibid., in press.

\section{References}

Ginzburg, V. L., Kirzhnits, D. A., and Liubushin, A. A. : 1971, Zh. Eksp. Teor. Fiz. 60, 451 (in Russian). Harrison, E. R.: 1970, Phys. Rev. D1, 2726.

Parker, L.: 1969, Phys. Rev. 183, 1057.

Peebles, P. J. E. and Yu, J. T.: 1970, Astrophys. J. 162, 815.

Zel'dovich, Ya. B.: 1961, Zh. Eksp. Teor. Fiz. 41, 1609.

Zel'dovich, Ya. B.: 1972, Monthly Notices Roy. Astron. Soc. 160, 18.

Zel'dovich, Ya. B.: 1973, Zh. Eksp. Teor. Fiz. 64, 58.

Zel'dovich, Ya. B. and Starobinsky, A. A.: 1971, Zh. Eksp. Teor. Fiz. 61, 2161.

\section{DISCUSSION}

Steigman: Is the particle production when passing through non-Friedmannian perturbations of the metric an artefact of improper joining of the solutions - or of the definition of particle number at early stages?

Zel'dovich: My belief is that anisotropic cosmological singularities should be prohibited due to particle formation.

Afterthought: The problem of what was before the singularity (and also if there was any 'before') was not mentioned at all at our Symposium. This does not mean that the problem does not exist!

Misner: You appear to assume that one should not speak of times less than $t_{\text {Planck }}$ because theories of quantum gravity are not fully developed. But model theories, as in my talk, suggest that, for instance, a quantum language may be developed in which analogues of Kasner-like or more general singularities are still meaningful and even inevitable as precursors of the pair creation epochs. I think one should speak of these sub-Planck times in order to let the language develop, and not exclude the possibility that only in this language - implicit in current physical principles - would the initial conditions of the Universe seem simple.

Icke: (a) Could the particle creation take the form of an instability, namely: curvature creates particle oairs which in turn create curvature and so forth?

(b) I did not understand where the conservation laws of physics are hidden in your formalism.

Zel'dovich: (a) The energy stress tensor of created particles acts on the metric in a direction which restores isotropy. So it is a kind of overstability, or a kind of viscous damping, and not instability.

(b) The exact conservation laws concern charges (baryonic, electric, leptonic) but not numbers of particles - for example, particle creation, $e^{+}+e^{-}$pairs or photon emission. These charge laws are fulfilled. The particle conservation laws are only approximate due to slow frequency variation, for example, in the Hubble expansion - and they are violated.

Steigman: Since you use such a stiff equation of state $p=\varepsilon$, all of the energy is in the field. Is it then possible to support fluctuations of any size (even as small as $10^{-4}$ ) in such a case?

Zel'dovich: The exact value of the energy depends on the position of baryons, and the number of degrees of freedom is $3 n$ as in Debye theory. The highbrow formulation of the cutoff for wave vectors is $4 \pi \int_{0}^{k} k^{2} \times$ $\times \mathrm{d} k=n$. The distribution of baryons over space in a cold baryonic fluid in the limiting stiff case is not clear (is it a Fermi-liquid or a crystalline state?) because of the long range of forces. Your question is an important hint for further study. In addition to a short letter in Monthly Notices, which I have already mentioned, a full version is now in print in JETP, 1973. 